\title{
BMJ Open Effect of involving certified healthcare assistants in primary care in Germany: a cross-sectional study
}

\author{
Jonas D Senft (1) , Michel Wensing, Regina Poss-Doering, Joachim Szecsenyi, \\ Gunter Laux
}

To cite: Senft JD, Wensing M, Poss-Doering $\mathrm{R}$, et al. Effect of involving certified healthcare assistants in primary care in Germany: a crosssectional study. BMJ Open 2019;9:e033325. doi:10.1136/ bmjopen-2019-033325

- Prepublication history for this paper is available online. To view these files, please visit the journal online (http://dx.doi org/10.1136/bmjopen-2019033325).

Received 08 August 2019 Revised 23 November 2019 Accepted 25 November 2019

Check for updates

(C) Author(s) (or their employer(s)) 2019. Re-use permitted under CC BY-NC. No commercial re-use. See rights and permissions. Published by BMJ.

Department of General Practice and Health Services Research, University Hospital Heidelberg, Heidelberg, Germany

Correspondence to Dr Jonas D Senft; jonas.senft@med.uniheidelberg.de

\section{ABSTRACT}

Objectives Growing prevalence of chronic diseases and limited resources are the key challenges for future healthcare. As a promising approach to maintain high-quality primary care, non-physician healthcare professionals have been trained to broaden qualifications and responsibilities. This study aimed to assess the influence of involving certified healthcare assistants (HCAs, German: Versorgungsassistent/in in der Hausarztpraxis) on quality and efficacy of primary care in Germany.

Design Cross-sectional study.

Setting Primary care.

Participants Patients insured by the Allgemeine Ortskrankenkasse (AOK) statutory health insurer (AOK, Baden-Wuerttemberg, Germany).

Interventions Since 2008 practice assistants in Germany can enhance their professional education to become certified HCAs.

Primary and secondary outcome measures Claims data related to patients treated in practices employing at least one HCA were compared with data from practices not employing HCAs to determine frequency of consultations, hospital admissions and readmissions. Economic analysis comprised hospitalisation costs, prescriptions of follow-on drugs and outpatient medication costs.

Results A total of 397493 patients were treated in HCA practices, 463730 patients attended to non-HCA practices. Patients in HCA practices had an $8.2 \%$ lower rate of specialist consultations $(p<0.0001)$, a $4.0 \%$ lower rate of hospitalisations $(p<0.0001)$, a $3.5 \%$ lower rate of readmissions ( $p=0.0463)$, a $14.2 \%$ lower rate of followon drug prescriptions $(p<0.0001)$ and $4.7 \%$ lower costs of total medication $(p<0.0001)$. No difference was found regarding the consultation rate of general practitioners and hospital costs.

Conclusions For the first time, this high-volume claims data analysis showed that involving HCAs in primary care in Germany is associated with a reduction in hospital admissions, specialist consultations and medication costs. Consequently, broadening qualifications may be a successful strategy not only to share physicians' work load but to improve quality and efficacy in primary care to meet future challenges. Future studies may explore specific tasks to be shared with non-physician workforces and standardisation of the professional role.

\section{Strengths and limitations of this study}

- This is the first high-volume claims data analysis assessing the effect of involving higher qualified practice assistants on quality and efficacy healthcare indicators in Germany.

- The analysis is performed on a comprehensive sample of data of 1 year covering 861223 patients.

- Statistical adjustment was possible for relevant patient-sided factors like patients' age and morbidity, nursing care level and structural factors like practice size, urbanisation and type (single or group practice).

- Due to the limitations given by the nature of claims data, further potentially relevant factors like educational level and experience of the staff were not available for this analysis.

- The professional role of healthcare assistants in Germany is not standardised, thus, limitations are given to the transferability of the intervention.

\section{INTRODUCTION}

All over the globe, providing access to highquality primary care is a challenge for healthcare systems. In the view of growing prevalence of chronic diseases and limited healthcare resources, physicians are confronted with increasing numbers of consultations while time is very limited. ${ }^{12}$

Particularly in times of evidence-based practice and growing use of treatment algorithms, time is needed to meet patient's individual preferences or circumstances, which are deciding factors for treatment success. ${ }^{34}$

Consequently, strategies are needed to maintain access to high-quality general practice. As a promising worldwide approach, highly qualified non-physician healthcare professionals, such as practice nurses in the USA or in Australia, are trained to take a more active role in primary care, particularly in treatment of patients with chronic diseases. ${ }^{5-8}$ For primary healthcare registered nurses and nurse practitioners in Canada, there is growing evidence that their involvement in 
practices is associated with health promotion, particularly in the management of chronic diseases. ${ }^{9-11}$

While qualified nurses are well integrated in primary care in other countries, in Germany so far there is no professional role for nurses in general medicine. On the other hand, non-academic workforces like practice or medical assistants have become increasingly involved into active patient care as they have been integrated into treatment monitoring or patient coaching for chronic diseases like diabetes, for example, in the USA. ${ }^{812}{ }^{13}$ In Germany, general practitioners (GPs) usually employ certified practice assistants, who absolved professional training for 3 years and traditionally performed clerical duties like reception and routine tasks, such as blood sampling or ECG recording. Since 2008 practical assistants may undergo an additional training programme of 200 hours to become certified as a so-called healthcare assistant (HCA) (German: 'Versorgungsassistent/in in der Hausarztpraxis' (VERAH)). HCAs are qualified to be closer involved in primary care delivery performing tasks such as team-based case management and monitoring of chronically ill patients, routine home visits and wound care. $^{7}$

However, to date there is only limited knowledge about the effect of broadening skills and responsibilities of nonphysician workforces on quality and efficacy of primary care. Recent RCTs did not find a beneficial effect of disease management programmes led by non-physician work forces on care indicators like hospitalisation rate or healthcare costs. ${ }^{14-16} \mathrm{~A}$ recent meta-analysis of 18 RCTs assessing the influence of nurses working as a substitute for physicians showed that nurse-led care may be equal in terms of health outcomes like control of diabetes and blood pressure and patient satisfaction. ${ }^{17}$

However, no evidence-based conclusion can be drawn currently with regard to the influence of involving higher qualified non-physician workforce on healthcare efficacy indicators like hospitalisation rate, specialist consultations and costs. Furthermore, common sample sizes of available RCTs may be underpowered to capture effects in this regard. The aim of this study was to assess the influence of involving certified HCAs on quality and efficacy of primary care in Germany. For this purpose, for the first time a high-volume claims data cross-sectional study was performed.

\section{METHODS}

\section{Study design}

A cross-sectional study was conducted. Claims data related to patients treated in general practices between 1 January and 31 December 2014 were supplied by the Allgemeine Ortskrankenkasse (AOK) statutory health insurance company (German: AOK, Baden-Wuerttemberg, Germany). Data of patients treated in practices employing at least one certified HCA were compared with data from practices not employing HCAs (non-HCA) to assess the influence of involving HCAs in primary care delivery

\section{Study population}

Secondary data related to patients insured by the AOK statutory health insurance company of BadenWuerttemberg, Germany, and participating in a specific primary care programme in Germany (GP-centred care; German: 'Hausarztzentrierte Versorgung' (HZV)) were eligible for data analysis. The federal state of BadenWuerttemberg has a population of about 10.7 million and AOK is the largest statutory health insurer with about 4 million insured persons. The HZV programme is a large-scale, legally stipulated care concept encouraging patients to enrol with a GP, aiming to strengthen primary care and to enhance healthcare for patients with chronic diseases and complex healthcare needs. ${ }^{18}$ Secondary patient data were included in the analysis, if patients met the following criteria: aged 18 years or older, living in Baden-Wuerttemberg, at least one visit to the primary care physician in the relevant year, no registration with other primary care contracts (eg, integrated care contracts), no interruptions of registration to $\mathrm{HZV}$ programme in the relevant year.

\section{Intervention}

Since 2008, practice assistants working in practices participating in the HZV programme in Germany can enhance their professional education by attending a standardised curriculum of 200 teaching units of theoretical and practical lessons. On mandatory examination, these practice assistants become state certified as HCA (German: VERAH). ${ }^{7}$ Besides routine tasks like blood sampling, ECG recording or spirometry, HCAs are thought to perform monitoring of chronically ill patients, prevention measures, routine home visits and wound care management.

\section{Data acquisition and outcome parameters}

Secondary patient data were recorded by the AOK state health insurance company for reimbursement purposes and continuous evaluation of the HZV programme. For the analysis, data were supplied by the AOK to the Department of General Practice and Health Services Research, University Hospital Heidelberg. Practices employing certified HCAs could be unambiguously identified since employment of HCAs is obligatorily reimbursed by state health insurance in the HZV programme. The claims data consisted of several data sets, containing particular information on patient care (eg, GP consultations, prescriptions and hospitalisations). These data could be linked on the basis of a unique patient identifier. Data linkage was performed by our research team using a relational database. Subjects cannot be identified, directly or through identifiers linked to the subjects. Data storage and extraction were performed with MySQL Community Server x64 (Oracle Corporation, Redwood Shores, California, USA). All national and institutional guidelines concerning data acquisition for retrospective analyses were followed at all times. 
The obtained data set comprised age, gender, diagnoses according to ICD-10 (International Statistical Classification of Diseases and Related Health Problems, 10th revision) coding as well as accounting data on consultations, prescribed medication and hospital stays.

To assess the effect of involving HCAs on quality and efficacy of primary care, the following outcome parameters were analysed: GP consultations, specialist consultations, hospital admissions, hospital readmissions within 4 weeks, hospitalisation costs, prescription of follow-on drugs and outpatient medication costs. The number of GP and specialist consultations per patient could be determined by the codes according to the system 'Einheitlicher Bewertungsmassstab' used for accounting of outpatient medical services in Germany. Number of hospital admissions and readmissions per patient as well as per-patient costs for hospitalisation in $€$ was determined by the recorded diagnosis-related groups codes used for reimbursement of inpatient medical services in Germany. The per-patient number of prescriptions of so-called follow-on drugs, patent-secured marginally altered pharmaceuticals with no benefit compared with the prototype drug according to evidence-based criteria, ${ }^{19}$ was determined by records of the central pharmaceutical numbers of prescribed medications ('Pharmazentralnummer'). Outpatient medication costs per patient in $€$ could be determined by accounting data for prescriptions reimbursed by the AOK state health insurance.

\section{Statistical analysis}

The full sample of available claims data was used for the analysis. In order to calculate frequencies, rates and percentages, we used SAS PROC SQL. In order to assess the adjusted outcomes of interest, we used SAS PROC GENMOD (SAS V.9.4 x64, SAS Institute). There was no missing data within the underlying data set. If there was no utilisation for a particular patient, for example, no hospitalisation, this was denoted as ' 0 '. The following factors were selected ex ante for the adjustment of the comparison between groups: patient age, sex, morbidity according to Charlson Index, ${ }^{20}$ nursing home as residence, nursing care level (legally defined 4-point scale to assess need for nursing support), urbanisation (rural, urban), practice size (number of contacts in relevant period), type of practice (single, group). Comparison between groups was done by multivariable regression analysis, which the three-level clustering of patients, GPs and practices into account. Depending on the distribution of each outcome, linear regression, negativebinomial regression or Poisson regression models (for count data) were used. Since multiple hypotheses were tested in this analysis, the Bonferroni correction was used to compensate for multiple comparisons. For all analyses, results were considered statistically significant, if the $\mathrm{p}$ value was 0.05 or less.

\section{Patient and public involvement}

Exploring strategies to provide and maintain access to high-quality primary care is of public interest, particularly
Table 1 Patient characteristics: data of patients treated in practices employing at least one HCA compared with practices not employing HCAs (non-HCA)

\begin{tabular}{llll}
\hline & HCA & Non-HCA & P value \\
\hline No of patients & 397493 & 463730 & \\
Male (N, \%) & $174415(43.9)$ & $200775(43.2)$ & $<0.0001$ \\
\hline Age & $56.9 \pm 18.5$ & $58.4 \pm 18.1$ & $<0.0001$ \\
\hline Charlson Index & $1.37 \pm 2.0$ & $1.38 \pm 1.98$ & $<0.0001$ \\
Care level (N) & & & $<0.0001$ \\
\hline No care: & 378919 & 442024 & \\
\multicolumn{1}{l}{ I } & 11186 & 13165 & \\
II & 5771 & 6765 & \\
\hline III & 1593 & 1751 & \\
\hline IV & 24 & 25 & \\
\hline
\end{tabular}

Continuous values are presented as mean \pm SD.

$\mathrm{HCA}$, healthcare assistant.

in the view of growing prevalence of chronic diseases and limited healthcare resources. Due to the retrospective study design based on an analysis of pseudonymised data, patients could not be identified, nor be informed or involved into this study. The public dissemination of the results is intended to be achieved by scientific publication.

\section{RESULTS}

A total of 861223 patients were evaluated in the observation period from 1 January to 31 December 2014. A total of 397493 patients were treated in practices involving at least one HCA to primary care (HCA group), 463730 patients were seen in practices, which did not employ HCAs (non-HCA group). Patients characteristics are shown in table 1 .

According to the adjusted analysis, patients in the HCA group had an $8.2 \%$ lower rate of specialist consultations $(\mathrm{p}<0.0001)$. Per-patient number of hospital admissions was $4.0 \%$ lower $(\mathrm{p}<0.0001)$ and number of hospital readmissions was $3.5 \%$ lower in the HCA group $(\mathrm{p}=0.0463)$. Prescriptions of follow-on drugs were $14.2 \%$ lower and total outpatient medication costs were $4.69 \%$ lower in the HCA-group, respectively $(\mathrm{p}<0.0001)$. No difference was found regarding the number of GP consultations and hospitalisation costs (table 2 ).

\section{DISCUSSION}

For the first time, this cross-sectional study assessed highvolume claims data to evaluate the influence of involving HCAs on quality and efficacy of primary care in Germany. The analysis of care-related data of 861223 patients showed a lower rate of hospital admissions, specialist consultations as well as lower outpatient medication costs when HCAs were part of the practice staff. Although the measured effect is low scaled, it is of high relevance for the development of future primary care concepts. From a 


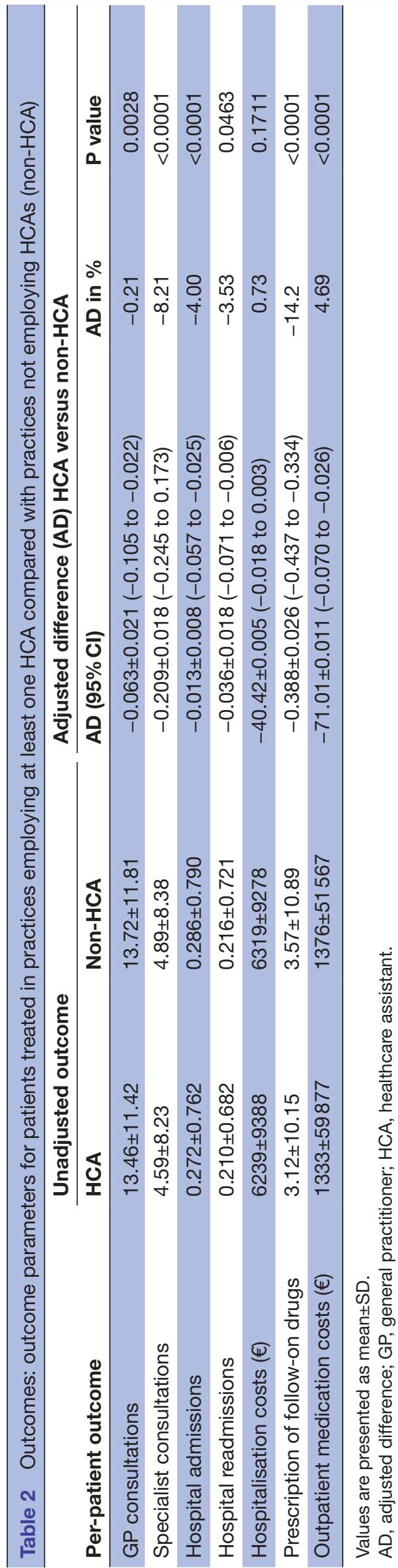

patient-centred view, avoiding hospitalisation or unnecessary medication may help to reduce patients' burden and morbidity due to hospital stay or pharmacological side effects. On the other hand, avoidable treatment will be not only a central determinant of quality, but a key cost factor for healthcare systems, which will be challenged by the rising prevalence of chronic diseases in the future.

The measured effect may be hypothesised to be due to an improved patient access to primary care. Either directly by being attended to by an HCA, or indirectly by improvement of workflow, patients may benefit from a higher quality and efficacy of care. Hospitalisations and specialist consultations may be avoided by a more intensive outpatient care facilitated by HCA involvement. Particularly, patients with chronic diseases may benefit from extended services like intense monitoring, education and reminders. ${ }^{21}$ And eventually, costs for prescriptions may be reduced by efficient management of medication regimen.

To date, knowledge about potential effects of involving higher qualified non-physician healthcare professionals in primary care is low. Several RCTs evaluating disease management programmes for chronic conditions like chronic pulmonary disease or heart failure involved practice assistants with enhanced educational training and responsibilities, however, did not prove an effect of these programmes on relevant care indicators. ${ }^{6} 1522$ A potential reason for this contrast to our findings may be an underpowered sample size postulating a reduction of avoidable hospitalisations up to $20 \%$. The results of our study show a much smaller effect with a reduction of $4 \%$ hospitalisations when HCAs were involved, which in our opinion is closer to reality in primary care. As a comparison, even in settings of complex disease management programmes for heart failure patients, low rates of reduction in all-cause hospitalisation are common when involving academically educated non-physician work forces and specialist physicians, with a range of up to $8 \%$ as a recent meta-analysis of 12 RCTs showed. ${ }^{23}$

Another relevant finding of this study is that the rate of GP consultations was only slightly reduced by $0.21 \%$ when HCAs were involved. This is noteworthy, since a distinct reduction of GP consultations might have been expected assuming that HCAs perform chosen routine tasks independently. One the other hand, this result may reflect that involvement of HCAs is not implemented as a one-way delegation or as a substitution for physician care as has been proposed for nurse-led care concepts, ${ }^{17}$ but more as a team interaction. However, no conclusion can be drawn by this study with regard to the specific role of HCAs within the practice staff. As a recent survey showed, in Germany, there is no firmly standardised professional role for HCAs. Performed tasks differ widely from simple patient assessment or basic wound care to tasks with substantial responsibility like emergency home visits, chronic care management or treatment of complex wounds. ${ }^{7}$ Eventually, the GP decides which tasks are performed by HCAs and to what extent they perform 
them independently. While this approach meets individual eligibility, more standardisation may be favourable to identify tasks to be shared in teams according to their effect on care quality and efficacy. Furthermore, it could help to reveal potential limitations, as found in nurse-led self-management programmes of COPD, which have been associated with higher airway-related mortality. ${ }^{15} 24$ A promising approach for a standardised involvement of HCAs certainly lies in chronic disease management programmes, which proved to be efficient for heart failure or asthma bronchiale. ${ }^{2325}{ }^{26}$ Furthermore, patient monitoring by HCAs could be supported using new IT-based methods such as web-based telemedical care, which has been shown to prevent hospital admissions and reduce all-cause mortality in heart failure patients. ${ }^{27}$ Finally, involving HCAs in standardised translational approaches after hospital release may be promising to reduce readmission rates. ${ }^{28} 29$

Limitations are given by the study design and the associated risk of confounding factors. Due to the nature of claims data, the parameters available for analysis were limited. The omission of practice details was an important element of the data protection contract for participating practices with the objective not to be identifiable by researchers. Thus, further potentially relevant factors such as educational level and experience of the staff or structural characteristics of the practices like equipment or procedural factors such as available diagnostics and treatment options, were not available for this analysis. Furthermore, the evaluation of relevant patient-reported outcomes such as quality of life was not possible in this analysis. On the other hand, we deliberately chose claims data for this analysis due to the high volume and statistical power necessary to assess the chosen outcomes. Furthermore, in our opinion, the available structural factors included in this analysis represent an appropriate and best possible adjustment for the measured outcomes.

This high-volume cross-sectional study showed that involving HCAs in primary care in Germany is associated with a reduction in hospital admissions, specialist consultations and overall medication costs. Consequently, broadening qualifications and responsibilities of non-physician work forces may be a successful strategy not only to alleviate physicians' workload, but to improve quality and efficacy of primary care to meet future healthcare challenges. Further studies should explore specific tasks to be shared with non-physician workforces and standardisation of the professional role.

Acknowledgements We would like to thank the statutory health insurance provider AOK Baden Wuerttemberg (Germany) for providing pseudonymised data for this analysis.

Contributors JDS: design and methods, writing the article; RP-D: data interpretation, revision of the article; MW: data interpretation, revision of the article; JS: design and methods, revision of the article; GL: design and methods, statistical analysis, revision of the article. All authors commented on the draft and approved the final version of the manuscript.

Funding The authors have not declared a specific grant for this research from any funding agency in the public, commercial or not-for-profit sectors.
Competing interests None declared.

\section{Patient consent for publication Not required.}

Ethics approval Ethical approval for this study was given by the local institutional Ethics Committee of the University Hospital Heidelberg (No. S-359/2013).

Provenance and peer review Not commissioned; externally peer reviewed.

Data availability statement Claims data used in this study were suplied by the AOK statutory health insurer (Allgemeine Ortskrankenkasse, Baden-Wuerttemberg, Germany) and are not available for third parties.

Open access This is an open access article distributed in accordance with the Creative Commons Attribution Non Commercial (CC BY-NC 4.0) license, which permits others to distribute, remix, adapt, build upon this work non-commercially, and license their derivative works on different terms, provided the original work is properly cited, appropriate credit is given, any changes made indicated, and the use is non-commercial. See: http://creativecommons.org/licenses/by-nc/4.0/.

ORCID iD

Jonas D Senft http://orcid.org/0000-0001-6989-950X

\section{REFERENCES}

1 Wolff JL, Starfield B, Anderson G. Prevalence, expenditures, and complications of multiple chronic conditions in the elderly. Arch Intern Med 2002;162:2269-76.

2 Fried TR, Tinetti ME, lannone L. Primary care clinicians' experiences with treatment decision making for older persons with multiple conditions. Arch Intern Med 2011;171:75-80.

3 Zolnierek KBH, Dimatteo MR. Physician communication and patient adherence to treatment: a meta-analysis. Med Care 2009;47:826-34

4 Kelley JM, Kraft-Todd G, Schapira L, et al. The influence of the patient-clinician relationship on healthcare outcomes: a systematic review and meta-analysis of randomized controlled trials. PLoS One 2014;9:e94207.

5 Ghorob A, Bodenheimer T. Sharing the care to improve access to primary care. N Engl J Med 2012;366:1955-7.

6 Freund T, Peters-Klimm F, Boyd CM, et al. Medical assistantbased care management for high-risk patients in small primary care practices: a cluster randomized clinical trial. Ann Intern Med 2016;164:323-30.

7 Mergenthal K, Beyer M, Gerlach FM, et al. Sharing responsibilities within the general practice team - a cross-sectional study of task delegation in Germany. PLoS One 2016;11:e0157248.

8 Freund T, Everett C, Griffiths P, et al. Skill mix, roles and remuneration in the primary care workforce: who are the healthcare professionals in the primary care teams across the world? Int J Nurs Stud 2015;52:727-43.

9 Donald F, Martin-Misener R, Bryant-Lukosius D, et al. The primary healthcare nurse practitioner role in Canada. Nurs Leadersh 2010;23:88-113.

10 Lukewich J, Edge DS, VanDenKerkhof E, et al. Association between registered nurse staffing and management outcomes of patients with type 2 diabetes within primary care: a cross-sectional linkage study. CMAJ Open 2016;4:E264-70.

11 Russell GM, Dahrouge S, Hogg W, et al. Managing chronic disease in Ontario primary care: the impact of organizational factors. Ann Fam Med 2009;7:309-18.

12 Halter M, Drennan V, Chattopadhyay K, et al. The contribution of physician assistants in primary care: a systematic review. BMC Health Serv Res 2013;13:223.

13 Ruggiero L, Riley BB, Hernandez R, et al. Medical assistant coaching to support diabetes self-care among low-income racial/ethnic minority populations: randomized controlled trial. West J Nurs Res 2014;36:1052-73.

14 Bekelman DB, Plomondon ME, Carey EP, et al. Primary results of the patient-centered disease management (PCDM) for heart failure study. JAMA Intern Med 2015;175:725-32.

15 Fan VS, Gaziano JM, Lew R, et al. A comprehensive care management program to prevent chronic obstructive pulmonary disease hospitalizations: a randomized, controlled trial. Ann Intern Med 2012;156:673-83.

16 Connor KI, Cheng EM, Barry F, et al. Randomized trial of care management to improve Parkinson disease care quality. Neurology 2019;92:e1831-42.

17 Laurant M, van der Biezen M, Wijers N, et al. Nurses as substitutes for doctors in primary care. Cochrane Database Syst Rev 2018;7. 
18 Laux G, Kaufmann-Kolle P, Bauer E, et al. [Evaluation of family doctor centred medical care based on AOK routine data in BadenWürttemberg]. Z Evid Fortbild Qual Gesundhwes 2013;107:372-8.

19 Fricke U, Hein L, Schwabe U. Neue Arzneimittel 2016. In: Schwabe U, Paffrath D, Ludwig W-D, et al, eds. Arzneiverordnungs-Report 2017. Berlin, Heidelberg: Springer Berlin Heidelberg, 2017: 55-135.

20 Sundararajan V, Henderson T, Perry C, et al. New ICD-10 version of the Charlson comorbidity index predicted in-hospital mortality. $J$ Clin Epidemiol 2004;57:1288-94.

21 Freund T, Mahler C, Erler A, et al. Identification of patients likely to benefit from care management programs. Am J Manag Care 2011;17:345-52.

22 Siebenhofer A, Ulrich L-R, Mergenthal K, et al. Primary care management for patients receiving long-term antithrombotic treatment: a cluster-randomized controlled trial. PLoS One 2019;14:e0209366.

23 Takeda A, Martin N, Taylor RS, et al. Disease management interventions for heart failure. Cochrane Database Syst Rev 2019;1.

24 Lenferink A, Brusse-Keizer M, van der Valk PD, et al. Selfmanagement interventions including action plans for exacerbations versus usual care in patients with chronic obstructive pulmonary disease. Cochrane Database Syst Rev 2017;8.

25 Smith SM, Soubhi H, Fortin M, et al. Interventions for improving outcomes in patients with multimorbidity in primary care and community settings. Cochrane Database Syst Rev 2012.

26 Peytremann-Bridevaux I, Arditi C, Gex G, et al. Chronic disease management programmes for adults with asthma. Cochrane Database Syst Rev 2015.

27 Koehler F, Koehler K, Deckwart O, et al. Efficacy of telemedical interventional management in patients with heart failure (TIM-HF2): a randomised, controlled, parallel-group, unmasked trial. Lancet 2018;392:1047-57.

28 Hesselink G, Schoonhoven L, Barach P, et al. Improving patient handovers from hospital to primary care: a systematic review. Ann Intern Med 2012;157:417-28.

29 Braet A, Weltens C, Sermeus W. Effectiveness of discharge interventions from hospital to home on hospital readmissions: a systematic review. JBI Database System Rev Implement Rep 2016;14:106-73. 